\title{
Covid, Psychology and Suicide Risk
}

Rory O'Connor

Prof. Rory O'Connor has a long-standing interest in suicide research and prevention; he has been working in this area since 1994. He is Professor of Health Psychology at the University of Glasgow in Scotland and Past President of the International Academy of Suicide Research. Rory leads the Suicidal Behaviour Research Laboratory at the University of Glasgow, one of the leading suicide and self-harm research groups in the UK. He has published widely in the field of suicide and selfharm, particularly on the psychological processes which precipitate suicidal behaviour and self-harm. He also acts as an advisor to a wide range of national and international organisations, including national governments, in the areas of suicide and self-harm.

Abstract. Since the onset of the Covid pandemic there has been concern about the short, medium and long-term impacts on population mental health. In this talk, drawing from the UK Covid-19 Mental Health and Wellbeing Study, as well as the wider research literature, I will describe the changes in mental health and wellbeing over the course of the pandemic. I will also outline some other ongoing work on suicide prevention research as well as discussing the opportunities to mitigate the risk of the longer term impacts of Covid-19 and its consequences on suicide risk. 